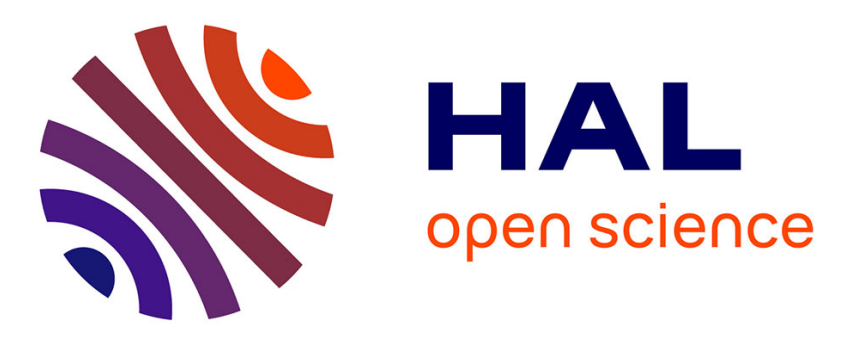

\title{
Integrated Production and Predictive Maintenance Planning based on Prognostic Information
}

Omar Bougacha, Christophe Varnier, Noureddine Zerhouni, S Hajri-Gabouj

\section{To cite this version:}

Omar Bougacha, Christophe Varnier, Noureddine Zerhouni, S Hajri-Gabouj. Integrated Production and Predictive Maintenance Planning based on Prognostic Information. International Conference on Advanced Systems and Electric Technologies, Mar 2019, Hammameth, Tunisia. hal-03107816

\section{HAL Id: hal-03107816 https://hal.science/hal-03107816}

Submitted on 12 Jan 2021

HAL is a multi-disciplinary open access archive for the deposit and dissemination of scientific research documents, whether they are published or not. The documents may come from teaching and research institutions in France or abroad, or from public or private research centers.
L'archive ouverte pluridisciplinaire HAL, est destinée au dépôt et à la diffusion de documents scientifiques de niveau recherche, publiés ou non, émanant des établissements d'enseignement et de recherche français ou étrangers, des laboratoires publics ou privés. 


\section{Integrated Production and Predictive Maintenance Planning Based on Prognostic Information}

\author{
Omar Bougacha, Christophe Varnier, Noureddine Zerhouni \\ Département AS2M \\ Institut FEMTO-ST \\ Université de Bourgogne Franche-Comté, ENSMM, CNRS \\ Besançon, France \\ firstname.lastname@femto-st.fr
}

\author{
Sonia Hajri-Gabouj \\ Laboratoire d'Informatique \\ pour les Systèmes Industriels (LISI) \\ Institut National des Sciences Appliquées et de Technologie \\ Université de Carthage \\ Tunis, Tunisie \\ sonia.gabouj@insat.rnu.tn
}

\begin{abstract}
This paper address the problem of scheduling production and maintenance operation in predictive maintenance context. It proposes a contribution in the decision making phase of the prognostic and health management framework. The prognostics and decision processes are merged and an ant colony optimization approach for finding the sequence of decisions that optimizes the benefits of a production system is developed. A case study on a single machine composed of several components where machine can have several usage profiles. The results show that our approach surpasses classical condition based maintenance policy.
\end{abstract}

Keywords-Production and Maintenance Scheduling, Predictive Maintenance, Prognostic Information, Remaining Useful Life, Ant Colony Optimization

\section{INTRODUCTION}

Nowadays, the industry sector is experiencing an economic pressures due to the highly competitive environment driven by the market requirements. Which motivated the manufacturers to optimize their process in term of time, cost and quality. In order to do so, companies are focusing on production and maintenance activities improvement. Therefore, one can find considerable number of research work on these two major activities of the industry sector.

In the last decades, production scheduling, an important task of manufacturing, has been extensively studied. One can find several works in the literature that aims to optimize the production planning of manufacturing system. One can refer to [1], [2], [3] and [4], for more information about production scheduling optimization and the various existing method to do so.

On the other hand, maintenance had its fair share of attention. Maintenance evolved from corrective maintenance where interventions are made after the failure of the system to systematic preventive maintenance where interventions are planned in advanced based on reliability information such as the Mean Time Between Failures. Not so long after, the development of condition monitoring technology made a break through in maintenance strategies, that resulted in the Condition-Based Maintenance (CBM). With CBM, the critical components of a system are identified, studied and supervised 978-1-7281-1317-3/19/\$31.00 @ 2019 IEEE to monitor their degradation level. Once the degradation level of a component or a system exceeds a predefined threshold a maintenance intervention is scheduled. literature reviews are proposed on maintenance scheduling in manufacturing context [5] and Condition-Based Maintenance [6]. Recently, the prediction techniques saw the light. Subsequently, they made one more upgrade to the maintenance policies by promoting the predictive maintenance [7]. Where, maintenance decision makers no longer settle for monitoring critical components and assessing their health status, but they predict the future states of the components and the system. Therefore it is possible to estimate how long a system will take to reach a failure state or a certain failure or event. The estimated duration is called Remaining Useful Life (RUL). The framework of monitoring, signal processing, health assessment, diagnostics, prognostics and decision making is called Prognostics and Health Management (PHM).

The maintenance planning and production scheduling are usually in conflict since they perform on the same resources. Although, undoubtedly, the fusion of these two activities will increase the agility of the manufacturing systems, they have always been optimized separately. One can still find a lot of works in literature that aims at optimizing one activity regardless of the other, yet in the last decade, one can easily notice the emergence of the optimization of maintenance and production scheduling simultaneously. Kaabi et al. [8], developed four heuristics to minimize the total tardiness of jobs on a single machine subject to systematic preventive maintenance. Benbouzid et al. [9], presented a sequential strategy and an integrated one to schedule maintenance and production activities in a flow shop. Few years later, Khelifati and Benbouzid-Sitayeb [10] proposed a multi-objective genetic algorithm to solve the same problem in a job-shop workshop. Najid et al. [11], optimized the joint scheduling of production and systematic maintenance for a multiple objects lot-sizing problem using a mixed integer linear programming. Fitouhi and Nourelfath [12], integrated noncyclical preventive maintenance scheduling and production planning for multistate machines in order to minimize the total cost through a simulated annealing algorithm. 
The implementation of PHM techniques was the scope of several research papers in different decision making aspects, automatic control [13], logistics [14], mission assignment [15] and in particularly the mixed problem of production and maintenance scheduling. Pan et al. presented in [16], a mathematical programming formulation to minimize the maximum tardiness of a single machine by simultaneously scheduling production and predictive maintenance based on it's RUL. Varnier and Zerhouni in [17], solved the joint scheduling problem for a flow-shop workshop where the machines have two production modes that influences their degradation by optimizing the aggregated sum of makespan and maintenance delays. Fitouri et al. [18], proposed a heuristic to solve the problem of job shop production and predictive maintenance scheduling for a job shop based on prognostic information. The proposed approach aims at minimizing the makespan and the total cost of maintenance. Ladj et al. [19] solved the integrated production and maintenance scheduling for a multi-functional single machine, in order to minimize the total maintenance cost, where each job is characterized by a degradation level. Later, Ladj et al., in [20], presented a hybrid of variable neighbor search and fuzzy logic to solve predictive maintenance and production scheduling for a permutation flow shop where each of the considered machines has its deterioration level, remaining useful life and a future degradation value when processing each kind of job. Liu et al. [21], used the health states and the RUL of a single machine to jointly schedule production activities and maintenance actions in the aim of minimizing the total cost. Bencheikh et al., presented in [22], a process to solve the joint problem for a multi-machine workshop using multi-agent systems. The agents are used to model the machines and the tasks. As the agents negotiate the schedule of production and maintenance based of the health states of the machines. Cheng et al. [23], presented a methodology to solve the problem of scheduling production and maintenance on a single machine while including the quality management of the produced parts.

In the previous studies, either a unique estimation of the RUL or the health indicators of the machines was performed and the obtained values is compared against a threshold to schedule maintenance regardless of the tasks being processed. Or the production tasks have a predetermined value of the amount of RUL they are consuming. However, the degradation of the machine under a new task depends of the initial degradation of the machine and the operational profile used to perform the task. This paper presents a solution for the problem of jointly scheduling maintenance and production tasks on a single machine while dynamically incorporating the new available health indicators. A modified Ant Colony Optimization (ACO) algorithm is used to solve this problem in order to maximize the total profit of the workshop over a simulation period.

The remaining of the paper is organized as follows. In section II, the integrated scheduling problem is detailed. A modified ACO algorithm and a classic process of joint scheduling using Moore algorithm and CBM are presented in section III. The developed algorithms are tested on a case study and the obtained results are discussed in section IV. Finally a conclusion of the works and some future works are given in the last section.

\section{Problem Statement}

\section{A. Machine Model}

In order to implement Prognostics and Health management techniques in the resolution of the joint problem of scheduling maintenance and production, one needs to define the problem in a PHM context. Thus we are going to consider for this problem a single machine that is capable to produce $N$ types of products (where these products are different in the production process, type of material, etc...) and with $M$ different operational profiles. An operational profile can be considered as a speed, or production rate. The considered machine is composed of $L$ components. Interactions between components can be divided into three categories, economic, structural and stochastic dependencies [24]. In this problem only structural dependencies will be considered between the components of the system, in a way that if one component fails the whole system fails. Each component of the system is supposed to be subject to a degradation only when the machine is producing. The degradation of a component is also supposed to be influenced by the task the machine is processing and by the profile of execution of the task. For simulation purposes, we used an exponential function as a degradation model for each component. Thus the degradation of component $l$ while producing product $n$ with the profile $j$ is given in equation 1 .

$$
D_{l, n, j}(x)=a_{l} \times\left(\exp ^{b_{l} \times S_{j} \times \rho_{n, l} \times x}-1\right)
$$

Where $a_{l}$ and $b_{l}$ are two parameters that defines the scale of the exponential function for a component $l . S_{j}$ is the coefficient that reflects the influence of production profile $j$ on the degradation of the component. $\rho_{n, l}$ reflects the severity of producing product $n$ on the degradation of the component.

The RUL of component $l$ is defined as the time left where component $l$ can still produce before its degradation level reached a failure threshold noted $t h_{l}$.

\section{B. Production Problem}

The production problem consists of the scheduling of $K$ production order. Each production order $k$ has a release date $r_{k}$, a due date $d_{k}$, a deadline $D_{k}$ and a quantity $Q_{k}$ of a product type to be manufactured. The machine is able to produce at most one production order at a moment. The production orders are available at their release date and should be finished before their due date. If the order is done after its due date, then a penalty will be paid by the company for tardiness, and the amount of the penalty is independent to the duration of the tardiness. Any order that exceeds its deadline is considered as a lost opportunity.

The scheduled order $k$ with a chosen production profile $j$ is defined in this work as a local decision of production. Thus 
such a local decision has a cost and an estimated gain that are described in equation 2 and 3 respectively.

$$
\begin{gathered}
C_{p}(k)=Q_{k} * C_{n, j}+u_{k} * Q_{k} * L P_{n} \\
G_{p}(k)=Q_{k} * P_{n}
\end{gathered}
$$

With $C_{n, j}$ is the price of producing one unit of product $n$ with production profile $j, u_{k}$ is equal to 1 if the order $k$ is finished late otherwise $0, L P_{n}$ is a tardiness penalty for the product $n$ and $P_{n}$ is the selling price of one unit of product $n$.

\section{Maintenance Problem}

To guarantee the availability of the machine and its reliability, some maintenance activities needs to be scheduled from time to time to replace the compromised components. Once a component is maintained it is considered to be in an "as good as new" state. The scheduling of a maintenance activity for a group of components (noted $P M$ ) is defined in this work as a local decision of maintenance. The maintenance activity does not have a direct effect on the estimated gain. Therefore, the gain of a maintenance local decision $G_{m}$ is equal to zero. On the other hand, the cost of a maintenance local decision is defined in equation 4 .

$$
C_{m}(l \in P M)=\sum_{l \in P M}\left(M_{l}+P R U L_{l} * R E P_{l}\right)
$$

With :

$$
R E P_{l}=\left\{\begin{array}{cc}
R U L_{t h}-R U L_{l} & \text { if } R U L_{t h}<R U L_{l} \\
0 & \text { otherwise }
\end{array}\right.
$$

Where $R U L_{l}$ is the RUL of component $l, R U L_{t h}$ is a predefined threshold for the RUL of component $l$ and $P R U L_{l}$ is a penalty on the not used portion of the maintained component.

\section{Production and Maintenance Scheduling Strategy}

The objective behind this application is to find the suitable compromise between the production and the maintenance activities. As a matter of fact, the aim is to establish a settlement between producing with different production profiles and maintaining the system to maximize the factory benefits over a simulation horizon $S H$. The simulation horizon will be divided into smaller decision horizon called steps. At each step the joint schedule of maintenance and production activity well be built out of the local decisions of production and maintenance. The objective function can be described by equation 6.

$$
\max \sum_{i \in S H} B_{i}=\max \sum_{i \in S H} G_{i}-C_{i}
$$

With $B_{i}$ the benefits of the $i^{t h}$ step and $C_{i}$ and $G_{i}$ are respectively the cost and the gain of the $i^{\text {th }}$ step as described in equation 7 and 8.

$$
\begin{gathered}
C_{i}=\sum C_{p}(k)+\sum C_{m}+L_{o p} *\left(A_{i}-U_{i}-M_{i}\right) \\
G_{i}=\sum G_{p}(k)
\end{gathered}
$$

With: $L_{o p}$ is a penalty on the time where the machine is capable of producing but it is idle for the lack of a production order. $A_{i}, U_{i}$ and $M_{i}$ are respectively the available time for production, the time where the machine is used to produce and the time spent in maintenance in the $i^{t h}$ step.

\section{RESOLUTION}

Most of the works in literature that implemented health management techniques into the resolution of the joint scheduling of production and maintenance, either estimated only one RUL value or supposed that the effects of the decisions on the degradation of the system is known in advance. Unlike those works, the present paper treats the decision making process of the PHM framework as interdependent with the prognostic process. In other words, this work consider the effect of a selected decision on the system health and the new possible decision that can be introduced by a change in the estimated RUL.

The idea is to adapt the PHM framework to build decision iteratively over a decision horizon. For this, suppose that the prognostic module can be equipped with two different algorithm : (i) the first algorithm will be used to make long-term predictions to estimate the RUL value of the system, (ii) the second algorithm will be used to make short-term predictions to estimate the future state of the system while executing a local decision (i.e. the production of a job or the maintenance of some components). To guarantee the building aspect of the decision, a decision building module is introduced with two main operations : (i) finding all possible jobs to schedule at time $t$, (ii) evaluate the outcome of the short-term prognostics and schedule one job of the list of feasible decisions. This proposed framework is presented in figure 1.

In figure 1, the decision building loop is represented with the dashed arrows, while the physical loop of decision making is represented by the full arrows. At each execution of the PHM process to make an integrated schedule of production and maintenance activities, the physical loop is executed once while the decision building loop is executed several times depending on the parameters used to control the loop. Actually for the decision building loop the process is the following: the short term prognostics state is updated to match the state of the real system, then the decision building module creates a list of all possible decisions, the items on this list are evaluated in the short term prognostics algorithm and a new list of the feasible decisions is created. At last the decision building selects one decision of the feasible list and updates the state of the short term prognostics and so on until the duration of the decision horizon is reached.

\section{A. Ant Colony Optimization}

To save the construction aspect of the decision, a modified Ant Colony Optimization (ACO) algorithm is set in place. The ACO [25], is modified to incorporate the shortterm prognostics and some technicalities about the decision building process. The modified ACO proposed is presented in algorithm 1. Actually the decision building loop presented in 


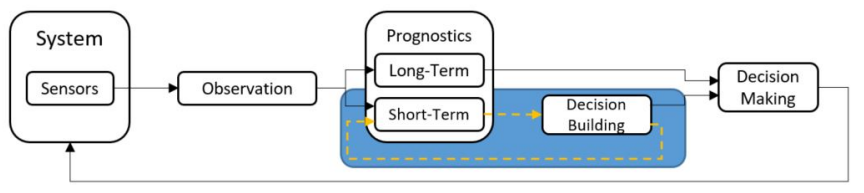

Fig. 1. PHM framework for joint scheduling

figure 1 is implemented in each ant of the colony in a way that every ant is equipped with its own short-term prognostics and the ant itself has the role of the decision building module. At each execution of the decision building process, each ant of the colony is set to build a schedule. Once all ants have built their schedules the best schedule is selected and the pheromones quantities on the jobs are updated and with that a new cycle of construction is launched until the number limit of cycles is reached.

The selection of the local decision of the list of the feasible decisions is made with a probability selection where each of the local decision has a selection probability defined by equation 9 .

$$
p_{k}(t)=\frac{\left(q_{k}\right)^{\alpha} *\left(\frac{1}{C(k)}\right)^{\beta_{1}} * G(k)^{\beta_{2}}}{\sum_{i \in \operatorname{List}(t)}\left(q_{i}\right)^{\alpha} *\left(\frac{1}{C(i)}\right)^{\beta_{1}} * G(i)^{\beta_{2}}}
$$

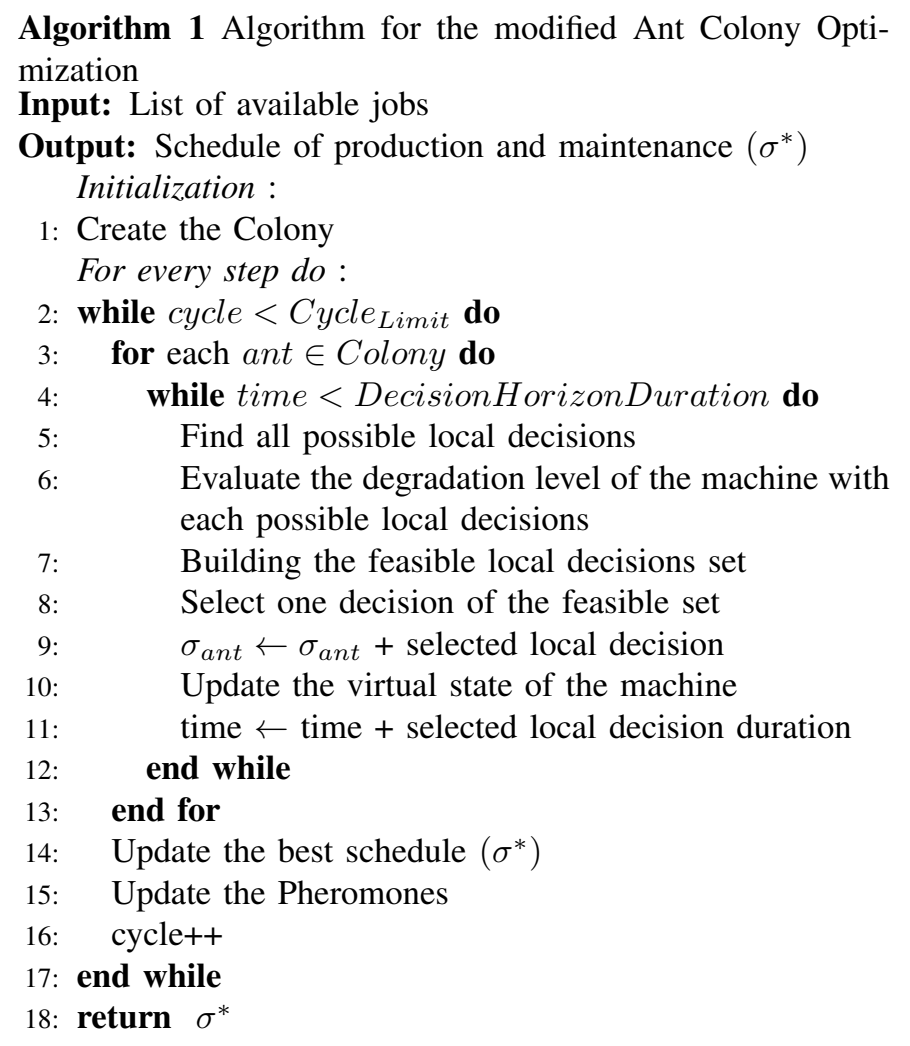

\section{B. Moore Algorithm with Condition Based Maintenance}

The proposed ACO is compared to a classical method of joint optimization of production and maintenance scheduling without implementing the health management concept. For this, all the equation recently presented will be the same except for equation 4 where the RUL is no longer computed.

The maximization of the benefits is obtained by maximizing the gain and minimizing the total cost. Thus, we focus here on the minimization of the total cost by choosing to minimize the cost of tardiness. Therefore we choose the Moore Algorithm as defined in [26], for it is known to minimize the number of tardy jobs.

The Moore algorithm is used to schedule the production The maintenance schedule is constructed using a Condition Based Maintenance (CBM) protocol. The resulting algorithm is presented in algorithm 2 and for rest of this paper this algorithm id denoted MCBM.

Algorithm 2 MCBM algorithm (Moore with Condition Based Maintenance)

Input: List of available jobs

Output: Schedule of production and maintenance $(\sigma)$

1: while $i<$ SimulationHorizon do

2: Inspection of the system

3: $\quad$ for $l \in$ Components do

4: $\quad$ if $D_{l}>$ Threshold failure $_{\text {then }}$

5: $\quad$ Schedule Corrective Maintenance

6: $\quad$ else if $D_{l}>$ Threshold preventive $_{\text {then }}$

7: $\quad$ Schedule Preventive Maintenance

8: $\quad$ end if

9: $\quad$ end for

10: Schedule the rest of the period $i$ with Moore Algorithm

11: i++

12: end while

13: return $\sigma$

\section{CASe Study}

\section{A. Numerical Example}

To apply the proposed approach, we consider the case of a single machine system. The machine has three components and it is able to produce four types of product with three different production profiles (low, medium and high speed).

- Different degradation dynamics were considered in making the test cases in order to study the influence of the degradation profile on the proposed approach.

- Different initial states of the system were considered, where each component has a different initial level of degradation.

- Three types of production orders were considered. The categories were built according to the quality of the demanded product; small tasks where $Q \in[10,50]$, medium tasks where $Q \in[30,100]$ and large tasks where $Q \in[100,200]$.

The combinations of the aforementioned parameters resulted in around 100 test cases. The results obtained in these test cases are presented in the next subsection.

\section{B. Results}

In this subsection, the obtained results are presented. Essentially three types of results are presented; (i) general results 


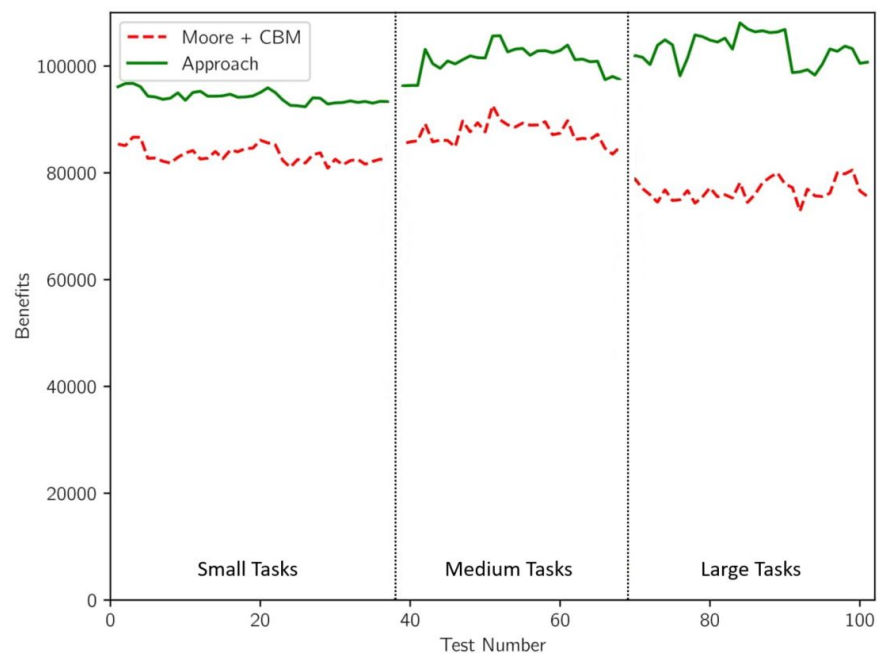

Fig. 2. Benefits of ACO vs MCBM

of the comparison between the ACO and the MCBM (ii) the results of the consistency of the ACO results (iii) the variability of the use of the different production profiles.

1) General results: Here, the results obtained by the implementation of PHM in the scheduling problem are compared to the benefits obtained by MCBM. Both of the results are presented in figure 2. As shown by the figure, the integration of prognostic information in the scheduling of production and maintenance guarantee higher benefits for the factory than using traditional approach like MCBM. Also, the bigger the tasks is the larger the improvement of the benefits using PHM are.

2) Robustness of the output: To study the robustness of the output schedule of the ACO algorithm, we run the same test case for 100 times and we compared the obtained results over one period. The obtained benefits are represented in figure 3 . Please note that for clarity purpose the range of the y-axis in figure 3 is from 2150 to 2300 . The maximum obtained benefits over this period is equal to 2292 , while the average benefits and the minimum benefits is equal to 2156 and 2281.94 respectively. Over the 100 run we had only 10 results below the average value where only 5 of them were equal to the minimum benefits. Plus the variation of the benefits between the worst result and the maximum result is less than $6 \%$ of the average benefits. On the other hand the benefits obtained for the same test case using the MCBM is equal to 1723 . Thus, the obtained results of the proposed approach is $20 \%$ more important than the benefits of the MCBM in the worst case. With $95 \%$ of the time, the capacity of the ACO algorithm to produce schedules with high benefits, we judge that our algorithm is robust toward the tackled problem.

3) Variability of the profile: One of the contributions of this paper is the assumption that the machine can function with different production profiles. The proposed algorithm is supposed to alter between the different profiles (i.e. speeds) to better manage the degradation of the components. Thus a

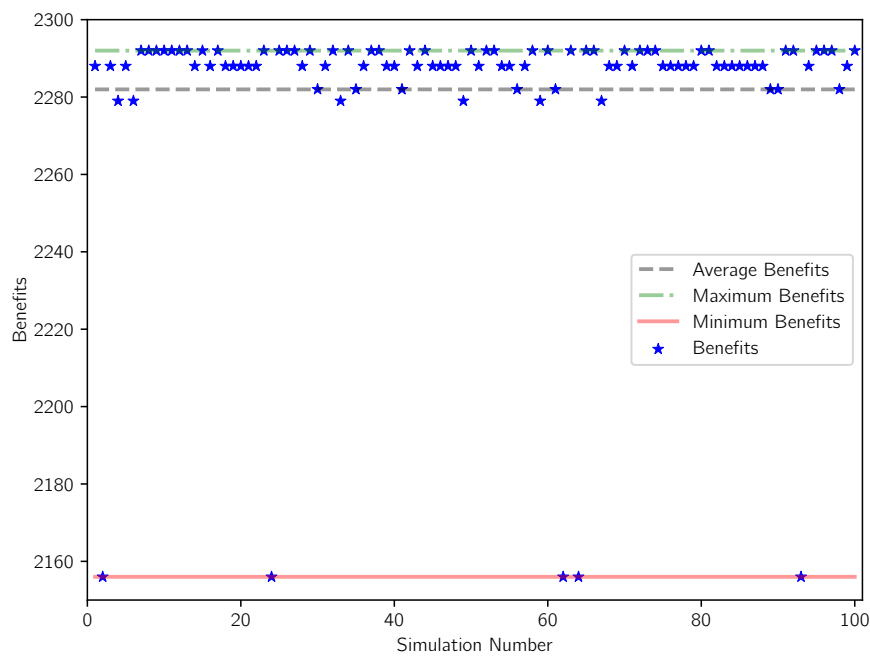

Fig. 3. Benefits of the same test case

study of the variability of the chosen profile to execute the various jobs is needed to evaluate this option. Table I present the percentage of each of the profiles in the case of the small, medium and large jobs.

Actually low speed production is characterized to be the least expensive production profile and the least degradation for the components. Thus the percentage of the use of this speed is the highest among the different test cases. Yet the use of low speed can effect the number of produced parts this explain the use of other production profiles. In the case of small jobs it is easier for the algorithm to find jobs that can fit the remaining time before the end of the period thus explain the low usage of the other speeds. When jobs tends to be bigger and with higher processing time the algorithm shift towards using more the high speed profile. By comparing the total produced parts between the proposed algorithm and the MCBM (table II), one can see that the frequent use of the low speed profile does not have a big influence on the total production. This can be explained that the machine is used more in production in the case of ACO so the time spent by the machine waiting for jobs is considerably less and that the time for maintenance is lower since the degradation of the machine is slower and no corrective maintenance is present.

TABLE I

VARIABILITY OF THE USED PROFILE

\begin{tabular}{|c|c|c|c|}
\hline Task Size & Low Speed & Medium Speed & High Speed \\
\hline Small & $74 \%$ & $18 \%$ & $8 \%$ \\
\hline Medium & $69 \%$ & $19 \%$ & $12 \%$ \\
\hline Large & $59 \%$ & $20 \%$ & $21 \%$ \\
\hline
\end{tabular}

\section{Conclusion}

In this work, we propose an ant colony optimization based algorithm to schedule maintenance and production on a single machine. The approach proposed is based on the integration of health management techniques in the process of scheduling, 
TABLE II

SiNGLE STEP PERFORMANCE COMPARISON

\begin{tabular}{|c|c|c|c|c|c|c|c|c|}
\hline \multirow{2}{*}{ Task Size } & \multicolumn{2}{|c|}{ Total Produced } & \multicolumn{2}{c|}{ U(\%) } & \multicolumn{2}{c|}{ M(\%) } & \multicolumn{2}{c|}{ L(\%) } \\
\cline { 2 - 9 } & ACO & MCBM & ACO & MCBM & ACO & MCBM & ACO & MCBM \\
\hline Small & 1475 & 1532 & 96.8 & 85 & 1.2 & 2 & 2 & 13 \\
\hline Medium & 1498 & 1574 & 97.9 & 87.5 & 1.5 & 2 & 1.6 & 10.5 \\
\hline Large & 1592 & 1533 & 97 & 85 & 1.2 & 2 & 1.8 & 13 \\
\hline
\end{tabular}

where the capability of the machine to perform the production tasks is assessed at every step of the construction of the schedule. Based on the predicted degradation level of the components, the machine defines its need for maintenance interventions. We show in this paper the importance of using prognostic information to help solve the conflicts between production and maintenance services. The obtained results were compared to a classic method of production scheduling combined with a classical periodic inspection condition based maintenance to prove the great capacity of the PHM framework. Still this work was based on strong assumptions like the usage of exponential function to model the degradation of the components and the availability of a high speed short term prognostic algorithm. For future works, we aim to integrate the uncertainties in the degradation estimation, some degradation models are available for such goal like the gamma process. Another future work is the use of the case based reasoning in the loop of decision building.

\section{REFERENCES}

[1] J. R. King and A. S. Spachis, "Heuristics for flow-shop scheduling," International Journal of Production Research, vol. 18, pp. 345-357, May 1980.

[2] S. C. Graves, "A Review of Production Scheduling," Operations Research, vol. 29, pp. 646-675, Aug. 1981.

[3] H. Aytug, M. Khouja, and F. E. Vergara, "Use of genetic algorithms to solve production and operations management problems: A review," International Journal of Production Research, vol. 41, pp. 3955-4009, Jan. 2003.

[4] J. Branke, S. Nguyen, C. W. Pickardt, and M. Zhang, "Automated Design of Production Scheduling Heuristics: A Review," IEEE Transactions on Evolutionary Computation, vol. 20, pp. 110-124, Feb. 2016.

[5] S.-H. Ding and S. Kamaruddin, "Maintenance policy optimizationliterature review and directions," The International Journal of Advanced Manufacturing Technology, vol. 76, pp. 1263-1283, Feb. 2015.

[6] R. Ahmad and S. Kamaruddin, "A review of condition-based maintenance decision-making," European J. of Industrial Engineering, vol. 6, no. 5 , p. $519,2012$.

[7] Z. M. Yang, D. Djurdjanovic, and J. Ni, "Maintenance scheduling in manufacturing systems based on predicted machine degradation," Journal of Intelligent Manufacturing, vol. 19, pp. 87-98, Feb. 2008.

[8] J. Kaabi, C. Varnier, and N. Zerhoni, "Heuristics for scheduling maintenance and production on a single machine," vol. vol.5, p. 5, IEEE, 2002.

[9] F. Benbouzid, C. Varnier, and N. Zerhouni, "Resolution of joint maintenance/production scheduling by sequential and integrated strategies," in Artificial Neural Nets Problem Solving Methods (J. Mira and J. R. lvarez, eds.), vol. 2687, pp. 782-789, Berlin, Heidelberg: Springer Berlin Heidelberg, 2003.

[10] S. L. Khelifati and F. Benbouzid-Sitayeb, "A Multi-Agent Scheduling Approach for the Joint Scheduling of Jobs and Maintenance Operations in the Flow Shop Sequencing Problem," in Computational Collective Intelligence. Technologies and Applications (P. Jdrzejowicz, N. T. Nguyen, and K. Hoang, eds.), vol. 6923, pp. 60-69, Berlin, Heidelberg: Springer Berlin Heidelberg, 2011.
[11] M. A. selsouli, N. Najib, A. Mohafid, and E. Aghezzaf, "An Integrated Production and Maintenance Planning Model with time windows and shortage cost," IFAC Proceedings Volumes, vol. 42, no. 4, pp. 822-827, 2009.

[12] M.-C. Fitouhi and M. Nourelfath, "Integrating noncyclical preventive maintenance scheduling and production planning for multi-state systems," Reliability Engineering \& System Safety, vol. 121, pp. 175-186, Jan. 2014.

[13] E. B. Pereira, R. K. H. Galvo, and T. Yoneyama, "Model predictive control using prognosis and health monitoring of actuators," in Industrial Electronics (ISIE), 2010 IEEE International Symposium on, pp. 237243, IEEE, 2010

[14] Y. Cui, J. Shi, and Z. Wang, "Discrete Event Logistics Systems (DELS) simulation modeling incorporating two-step Remaining Useful Life (RUL) estimation," Computers in Industry, vol. 72, pp. 68-81, Sept. 2015.

[15] I. P. de Medeiros, L. R. Rodrigues, R. Santos, E. H. Shiguemori, and C. L. N. Jnior, "PHM-based Multi-UAV task assignment," in Systems Conference (SysCon), 2014 8th Annual IEEE, pp. 42-49, IEEE, 2014.

[16] E. Pan, W. Liao, and L. Xi, "A joint model of production scheduling and predictive maintenance for minimizing job tardiness," The International Journal of Advanced Manufacturing Technology, vol. 60, pp. 1049-1061, June 2012.

[17] C. Varnier and N. Zerhouni, "Scheduling predictive maintenance in flowshop," pp. 1-6, IEEE, May 2012.

[18] C. Fitouri, N. Fnaiech, C. Varnier, F. Fnaiech, and N. Zerhouni, "A Decison-Making Approach for Job Shop Scheduling with Job Depending Degradation and PredictiveMaintenance," IFAC-PapersOnLine, vol. 49, no. 12 , pp. 1490-1495, 2016.

[19] A. Ladj, C. Varnier, F. B. S. Tayeb, and N. Zerhouni, "Exact and heuristic algorithms for post prognostic decision in a single multifunctional machine," International Journal of of Prognostics and Health Management, vol. 8 , no. 2, 2017.

[20] A. Ladj, F. Benbouzid-Si Tayeb, C. Varnier, A. A. Dridi, and N. Selmane, "A Hybrid of Variable Neighbor Search and Fuzzy Logic for the permutation flowshop scheduling problem with predictive maintenance," Procedia Computer Science, vol. 112, pp. 663-672, 2017.

[21] Q. Liu, M. Dong, F. Chen, W. Lv, and C. Ye, "Single-machine-based joint optimization of predictive maintenance planning and production scheduling," Robotics and Computer-Integrated Manufacturing, vol. 55, pp. 173-182, Feb. 2019.

[22] G. Bencheikh, A. Letouzey, and X. Desforges, "Process for joint scheduling based on health assessment of technical resources," IFACPapersOnLine, vol. 51, no. 24, pp. 192-199, 2018.

[23] G. Q. Cheng, B. H. Zhou, and L. Li, "Integrated production, quality control and condition-based maintenance for imperfect production systems," Reliability Engineering \& System Safety, vol. 175, pp. 251-264, July 2018.

[24] R. P. Nicolai and R. Dekker, Optimal Maintenance of Multi-Component Systems: A Review. In: Complex System Maintenance Handbook. Springer, London: Springer Series in Reliability Engineering, 2008.

[25] M. Dorigo and G. Di Caro, "Ant colony optimization: a new metaheuristic," pp. 1470-1477, IEEE, 1999.

[26] J. M. Moore, "An n Job, One Machine Sequencing Algorithm for Minimizing the Number of Late Jobs," Management Science, vol. 15, pp. 102-109, Sept. 1968. 